\title{
A SÍNDROME DA ESTAFA PROFISSIONAL EM MÉDICOS CANCEROLOGISTAS BRASILEIROS
}

\author{
Luciana Tomanik Cardozo de Melo Tucunduva, Ana Paula Garcia, fernanda Vilas Boas Prudente, Guilherme Centofanti, Carla Manzoni de Souza, \\ tatiana Alves Monteiro, flávio Augusto Henriques Vince, Eliana Sueco tibana Samano, Marina Sahade Gonçalves, Auro del Giglio* \\ Trabalho realizado no Instituto de Oncologia da Faculdade de Medicina do ABC
}

*Correspondência:

Av. Rebouças, 3357

Cep: $05410-040$

São Paulo, SP

\section{RESUMO}

OBJETIvos. A síndrome da estafa profissional (burnout) é um quadro caracterizado por três dimensões: exaustão emocional (EE), despersonalização (DP) e baixa realização pessoal (RP). Na área médica, os cancerologistas estão particularmente predispostos a esta síndrome. $\mathrm{O}$ objetivo deste estudo é avaliar sua prevalência entre cancerologistas brasileiros, correlacionando-a com dados demográficos e características de trabalho destes profissionais, avaliando também quais as suas sugestões para prevenção do quadro.

Métodos. Três questionários foram enviados aos 645 membros da Sociedade Brasileira de Cancerologia, por correio, e, após dez semanas, foram recebidas 136 respostas. Os questionários utilizados foram um de opinião, um geral e o inventário de Maslach de exaustão profissional, que avalia as supramencionadas dimensões separadamente, caracterizando-as em níveis leve, moderado ou grave.

Discussão. A taxa de resposta obtida foi $21 \%$. A síndrome foi observada em níveis moderados ou graves nas três dimensões em 15,7\% dos médicos. Para EE, 55,8\% apresentaram os níveis moderado ou grave. Para DP, esse número foi de $96,1 \%$ e, para RP, 23,4\%. Correlacionando o questionário Maslach com os dados demográficos, encontramos significância estatística entre prática de atividade física ou hobby e menores níveis de EE $(p=0,008)$ e trabalhar apenas em instituições privadas com maiores níveis de DP $(p=0,021)$. Os cancerologistas apontaram como alternativas mais relevantes na prevenção da síndrome menos burocracia (73,5\%) e limitação do número de pacientes atendidos (72,7\%).

ConcLusão. A síndrome da estafa profissional é freqüente entre médicos cancerologistas brasileiros, e outros estudos devem ser desenvolvidos para averiguar sua prevalência e prevenção em outras especialidades médicas.

UnITERMOs: Estafa profissional. Oncologia. Despersonalização.

\section{INTRODUÇão}

A síndrome da estafa profissional, também denominada síndrome do burnout, foi descrita pela primeira vez pelo psicólogo H.J. Freudenberger, no ano de 1974, para descrever um sentimento de fracasso e exaustão causado por um excessivo desgaste de energia, força e recursos ${ }^{1,2,3}$. Maslasch foi uma das pioneiras nos estudos empíricos sobre a estafa profissional, sendo a autora do primeiro trabalho publicado sobre o tema em $1986^{1,4,5}$.

A sindrome da estafa constitui um quadro bem definido, caracterizado por exaustão emocional, despersonalização e redução da realização pessoa $13,5,6,7,8$. A exaustão emocional representa o esgotamento dos recursos emocionais do indivíduo. É considerado o traço inicial da síndrome e decorre principalmente da sobrecarga e do conflito pessoal nas relações interpessoais ${ }^{1,29}$. A despersonalização é caracterizada pela insensibilidade emocional do profissional, que passa a tratar clientes e colegas como objetos ${ }^{1,2,9}$. Trata-se de um aspecto fundamental para caracterizar a síndrome de estafa (burnout), já que suas outras características podem ser encontradas nos quadros depressivos em geral2. Por fim, a redução da realização pessoal (ou sentimento de incompetência) revela uma auto-avaliação negativa associada à insatisfação e infelicidade com o trabalho $0^{1,2,9}$.
Os primeiros sentimentos negativos são direcionados aos desencadeantes do processo, ou seja, clientes e colegas de trabalho, posteriormente atingindo amigos e familiares e, por último, o próprio profissional. Sintomas físicos associados ao desgaste incluem cefaléia, alterações gastrointestinais e insônia, entre outros. As conseqüências da síndrome da estafa profissional podem ser graves, incluindo desmotivação, frustração, depressão e dependência de drogas. O desgaste se reflete também nas relações familiares (separações, maus tratos) e no trabalho, determinando diminuição importante do rendimento e aumento de absenteísmo ${ }^{10}$.

A estafa profissional pode ser observada em todas as profissões, principalmente naquelas que envolvem altos níveis de estresse, tais como controladores de tráfego aéreo, bombeiros e, particularmente, profissionais da área de saúde, como os médicos ${ }^{1,1,1,12}$. Isto se deve tanto às características inerentes à profissão - como convivio intenso com pacientes, intensidade das interações emocionais e a falta de tempo livre para lazer e férias - quanto às mudanças pelas quais a prática médica vem passando nos últimos 20 anos, que incluem progressivo declínio da autonomia profissional, diminuição do status social da profissão e aumento das pressões sofridas por estes profissionai ${ }^{5}$.

Globalmente, a estafa profissional afeta um em cada dois médicos, 
sendo um terço deles afetado de forma importante e um décimo de forma severa, com características irreversíveis ${ }^{5}$. Cerca de 40\% a 50\% dos médicos que trabalham com medicina de emergência e infectologia e $56 \%$ dos cancerologistas são acometidos pela síndrome ${ }^{12}$.

A cancerologia é uma especialidade particularmente estressante por diversas razões, dentre elas a maior exposição à morte e o conflito entre o objetivo de cura, para o qual todos os médicos são treinados, e a necessidade de prestar cuidados paliativos ${ }^{3}$. As principais causas observadas da estafa profissional entre os cancerologistas são: tempo insuficiente de férias, sentimento de impotência e expectativas irreais, raiva, frustração, dificuldade em lidar com perdas e assuntos relacionados a fontes pagadoras ${ }^{4}$.

Com base na importância da síndrome da estafa profissional e na escassez de estudos na literatura nacional a este respeito, investigamos sua prevalência e as características desta síndrome nos médicos cancerologistas brasileiros, avaliando suas opiniões quanto a possíveis formas de prevenção.

\section{Métodos}

Foi realizado um estudo observacional transversal descritivo, no período de novembro de 2003 a maio de 2004, envolvendo todos os médicos cancerologistas cadastrados na Sociedade Brasileira de Cancerologia.

Após a aprovação do protocolo pelo Comitê de Ética em Pesquisa da Faculdade de Medicina do ABC e autorização da Sociedade Brasileira de Cancerologia, foram enviados, pelo correio, questionários aos 645 membros, que compunham a amostra inicial.

Após dez semanas do envio dos três questionários, foram recebidos 136 respondidos e 14 adicionais não respondidos que retornaram ao remetente por mudança de endereço.

Cada envelope continha, além dos questionários, uma carta de apresentação explicando o desenho do estudo, e consideramos o reenvio dos questionários como a aprovação do consentimento informado.

\section{Os instrumentos utilizados foram:}

Questionário geral - composto por variáveis descritivas como idade, sexo, local de trabalho, tempo no posto atual, número de pacientes atendidos por dia, tempo de consulta, atividade física ou hobby, apoio familiar, carga horária semanal, realização de plantões e tempo de férias por $a n o^{5}$.

Questionário Maslach (Maslach Burnout Inventory) - composto por 22 afirmações sobre sentimentos e atitudes que englobam os três aspectos fundamentais da síndrome, divididos em três escalas de sete pontos, que variam de 0 a 63,14. Desta maneira, foram descritas, de forma independente, cada uma das dimensões que caracterizam a estafa profissional.

A exaustão emocional é avaliada por nove itens, a despersonalização por cinco e a realização pessoal por oito. As notas de coorte utilizadas foram as empregadas no estudo de Maslach ${ }^{14}$.

Para exaustão emocional, uma pontuação maior ou igual a 27 indica alto nível; de 19 a 26 nível moderado; e menor que 19 nível baixo. Para despersonalização, pontuações iguais ou maiores que 10 indicam alto nível, de seis a nove nível moderado e menores de seis nível baix $0^{3,5,10,14}$. A pontuação relacionada à realização pessoal vai em direção oposta às outras, uma vez que pontuações de zero a 33 indicam alto nível, de 34 a 39 nível moderado e maior ou igual a 40, baixo.

Por não haver consenso na literatura para interpretação do questionário Maslach, descrevemos os resultados segundo os critérios de Ramirez et al. e Grunfeld et al.. Os primeiros definem estafa profissional pela presença das três dimensões em nível grave, enquanto Grunfeld aceita a presença de uma em nível grave, independente de qual seja, para o diagnóstico da síndrome.

Questionário de opinião - formado por dez propostas para minimizar ou prevenir os efeitos da síndrome de desgaste profissional. Os participantes escolheram as cinco que consideraram como mais relevantes ${ }^{5}$.

Para a estatística descritiva e cálculo de intervalos de confiança (IC 95\%), utilizamos o programa Microsoft Excel (Windows Millenium Edition). Para a análise de estatística, utilizamos o programa NCSS 2000PASS 2000 (Utah, EUA). Na análise das variáveis discretas, utilizamos o teste do Qui-quadrado $\left(X^{2}\right)$ ou Fisher, quando necessário. Para comparação de médias, utilizamos o método Anova. Consideramos significativos valores de $p$ iguais ou menores que 0,05. Para análise multivariada, utilizamos também o programa NCSS 2000-PASS 2000 (Utah, EUA).

\section{Resultados}

A taxa de resposta obtida foi de 21 , I \%, uma vez que 136 respostas foram recebidas das 645 cartas enviadas. Se excluirmos da amostra os 14 questionários que retornaram por mudança de endereço, a taxa de resposta encontrada foi de $21,5 \%$.

Os dados demográficos referentes aos cancerologistas e as características de suas atividades profissionais estão descritos na Tabela I. A média de idade encontrada foi de 53,6 anos e a maioria da amostra foi composta por homens (82,8\%).

O tempo de trabalho na posição atual foi em média de 22,2 anos, e a maior parte trabalha em instituição privada e consultório particular (74,6\% e 68,6\%). Os participantes atendem em média 20 pacientes por dia, gastando com estes o tempo médio de 23 minutos por consulta. A maioria realiza atividade física ou hobby rotineiramente (56\%), conta com apoio familiar em seu cotidiano $(90,3 \%)$ e não costuma dar plantões com freqüência $(79,8 \%)$. O tempo de férias foi em média de 15 a 30 dias por ano (33,6\% e 32,8\%, respectivamente).

Quanto ao questionário de estafa profissional (Maslach), os resultados estão descritos na Tabela 2. Para a dimensão de exaustão emocional, o escore médio obtido foi de 21 ,4, considerado como nível moderado da síndrome, embora 44,2\% tenham apresentado nível baixo. Para despersonalização, a média foi de 9,7, e 59,4\% do grupo apresentaram nível moderado. Com relação à realização pessoal, o escore médio obtido foi de 42, e a maior parte dos entrevistados apresentou alta realização pessoal (76,6\%).

Avaliando-se as esferas moderadas e graves em conjunto para cada dimensão, 55,8\% apresentaram níveis moderados ou altos para exaustão emocional e 96, I \% para despersonalização. Para realização pessoal, foram observados em conjunto os níveis baixos e moderados em 23,4\% dos indivíduos que responderam ao questionário. Entretanto, tendo em vista apenas o nível grave (alto), 28, I \% apresentaram-no apenas em uma dimensão, $21 \%$ em duas e $3 \%$ nas três dimensões. 


\begin{tabular}{|c|c|}
\hline Variáveis & Resultados N (\%) \\
\hline Idade(média) & 53,6 \\
\hline \multicolumn{2}{|l|}{ Sexo } \\
\hline Feminino & $23(17,2 \%)$ \\
\hline Masculino & | | | (82,8\%) \\
\hline \multicolumn{2}{|l|}{ Local de trabalho* } \\
\hline Inst. pública & $74(55,2 \%)$ \\
\hline Inst. privada & $100(74,6 \%)$ \\
\hline Inst. de ensino & $46(34,3 \%)$ \\
\hline Consultório particular & $92(68,6 \%)$ \\
\hline Tempo de trabalho no posto atual (média) & 22,2 anos \\
\hline Média de atendimentos por dia & 20 pacientes \\
\hline Tempo médio de duração da consulta & $23 \mathrm{~min}$ \\
\hline \multicolumn{2}{|l|}{ Hobby ou atividade física } \\
\hline Sim & $75(56 \%)$ \\
\hline Não & $59(44 \%)$ \\
\hline \multicolumn{2}{|l|}{ Apoio familiar } \\
\hline $\mathrm{Sim}$ & $121(90,3 \%)$ \\
\hline Não & $13(9,7 \%)$ \\
\hline \multicolumn{2}{|l|}{ Carga horária semanal } \\
\hline $\mathrm{d} 40 \mathrm{~h}$ & $24(17,9 \%)$ \\
\hline 41 a $50 h$ & $32(23,9 \%)$ \\
\hline $51 \mathrm{a} 60 \mathrm{~h}$ & $42(31,3 \%)$ \\
\hline$>60 h$ & $36(26,9 \%)$ \\
\hline \multicolumn{2}{|l|}{ Plantões } \\
\hline Comfreqüência & $10(7,5 \%)$ \\
\hline Ás vezes & $17(16,7 \%)$ \\
\hline Não & $107(79,8 \%)$ \\
\hline \multicolumn{2}{|l|}{ Tempo de férias } \\
\hline pelo menos uma semana por ano & $16(\mid 1,9 \%)$ \\
\hline 15 dias por ano & $45(33,6 \%)$ \\
\hline 30 dias por ano & $44(32,8 \%)$ \\
\hline mais de 30 dias por ano & $4(3 \%)$ \\
\hline não tira férias já há um ano ou mais & $10(7,5 \%)$ \\
\hline não costuma tirar férias & $15(\mid 1,2 \%)$ \\
\hline
\end{tabular}

* Os resultados obtidos em relação ao local de trabalho somam mais de $100 \%$ devido ao fato de muitos oncologistas trabalharem em diferentes locais

De modo geral, 15,6\% dos entrevistados apresentaram níveis moderados ou graves para as três dimensões (ausência de níveis leves em todas as dimensões). Utilizando-se os critérios de Ramirez et al., $3 \%$ da amostra apresentaram estafa profissional. Já de acordo com Grunfeld et al., a síndrome foi observada em 52,3\% (Tabela 2).

Relacionando os dados demográficos com os resultados do questionário Maslach, em cada dimensão separadamente, foram encontradas correlações estatisticamente significativas entre prática de atividade física ou hobby com baixos níveis de cansaço emocional $(p=0,008)$, trabalhar exclusivamente em instituições públicas e menores níveis de exaustão emocional $(p=0,027)$ (Tabela 3).

Considerando o critério de Grunfeld, observamos correlação entre sexo feminino $(p=0,004)$ e ausência de apoio familiar em seu cotidiano $(p=0,025)$ com maior incidência da síndrome. Utilizando a análise multivariada, houve uma tendência para as mesmas variáveis $(p=0,07$ e $p=0,05)$. Utilizando o critério de Ramirez, não encontramos correlações estatisticamente significantes entre a síndrome da estafa profissional e as variáveis estudadas (Tabela 4).

\begin{tabular}{|c|c|}
\hline Critérios & Resultados \\
\hline $\begin{array}{l}\text { Dimensões isoladas } \\
\text { Exaustão emocional (escore médio) } \\
\text { Baixo } \\
\text { Moderado } \\
\text { Alto } \\
\text { Moderado e alto }\end{array}$ & $\begin{array}{c}21,4 \\
57(44,2 \%) \\
28(21,7 \%) \\
44(34,1 \%) \\
72(55,8 \%)\end{array}$ \\
\hline $\begin{array}{l}\text { Despersonalização(escore médio) } \\
\text { Baixo } \\
\text { Moderado } \\
\text { Alto } \\
\text { Moderado e alto }\end{array}$ & $\begin{array}{c}9,7 \\
5(3,9 \%) \\
76(59,4 \%) \\
47(36,7 \%) \\
123(96,1 \%)\end{array}$ \\
\hline $\begin{array}{l}\text { Realização pessoal (escore médio) } \\
\text { Baixo } \\
\text { Moderado } \\
\text { Alto } \\
\text { Baixo e moderado }\end{array}$ & $\begin{array}{c}42 \\
11(8,6 \%) \\
19(14,8 \%) \\
98(76,6 \%) \\
30(23,4 \%)\end{array}$ \\
\hline $\begin{array}{l}\text { Grunfeld et al. } \\
\text { Presença de: } \\
\text { Exaustão emocional em nível alto OU } \\
\text { Despersonalização em nível alto OU } \\
\text { Realização pessoal em nível baixo }\end{array}$ & $52,3 \%$ \\
\hline $\begin{array}{l}\text { Ramirez et al. } \\
\text { Presença de: } \\
\text { Exaustão emocional em nível alto E } \\
\text { Despersonalização em nível alto E } \\
\text { Realização pessoal em nível baixo }\end{array}$ & $3 \%$ \\
\hline
\end{tabular}

Em relação ao questionário de opinião, $73,5 \%$ consideraram alternativas mais relevantes menos burocracia, 72,7\% limitação do número de pacientes atendidos por dia, e 52,3\% maior quantidade e qualidade em formação continuada (Tabela 5).

\section{Discussão}

Este estudo é o primeiro na literatura nacional a avaliar a frequêencia da síndrome de estafa profissional (burnout). Trata-se de um tema relevante, a julgar pela alta frequeência desta síndrome entre oncologistas, reportada em estudos estrangeiros ${ }^{2}$. Encontramos em nosso meio pelo menos $28 \%$ dos entrevistados com pontuações referentes a uma das dimensões gravemente alteradas. Cerca de 15\% deles apresentaram alterações importantes simultaneamente nas três dimensões avaliadas.

Uma das importantes limitações em nosso estudo é a baixa taxa de resposta (2l\%) obtida. As taxas de resposta encontradas na literatura variaram entre $50,3 \%$ e $83 \%$. Nestes estudos, os autores estabeleceram um intervalo maior para recebimento da resposta e enviaram os questionários mais de uma vez (de duas a quatro vezes). Em nosso estudo, os questionários foram enviados apenas uma vez, e o período aguardado foi de dez semanas, o que pode justificar a menor taxa de resposta encontrada ${ }^{3,5,7,10,15}$. Entretanto, acreditamos que nossa amostragem foi aparentemente representativa, já que não houve diferenças significativas na distribuiçãao por estado de procedência dos questionários com a distribuição de todos os endereços dos membros da Sociedade Brasileira de Cancerologia por Estado (dados não reportados). 


\begin{tabular}{|c|c|c|c|c|}
\hline & $\begin{array}{l}\text { Exaustão } \\
\text { Emocional }\end{array}$ & Despersonalização & $\begin{array}{c}\text { Realização } \\
\text { pessoal }\end{array}$ & $\begin{array}{l}\text { Critério de } \\
\text { Grunfeld et al. }\end{array}$ \\
\hline Sexofeminino & 0,154463 & 0,921645 & 0,943452 & 0,004360 \\
\hline $\begin{array}{l}\text { Trabalharem } \\
\text { instituições públicas }\end{array}$ & 0,027493 & 0,717506 & 0,925803 & 0,947837 \\
\hline $\begin{array}{l}\text { Cultivar prática de } \\
\text { atividade física ou } \\
\text { hobby }\end{array}$ & 0,008172 & 0,527526 & 0,754927 & 0,288097 \\
\hline $\begin{array}{l}\text { Ausência de apoio } \\
\text { familliar }\end{array}$ & 0,533769 & 0,710118 & 0,471050 & 0,025768 \\
\hline
\end{tabular}

\begin{tabular}{|c|c|c|c|c|}
\hline \multirow[b]{2}{*}{$\begin{array}{l}\text { Variáveis } \\
\text { demográficas }\end{array}$} & \multicolumn{2}{|c|}{ Grunfeld } & \multicolumn{2}{|c|}{ Ramirez } \\
\hline & $\begin{array}{c}\text { Análise } \\
\text { univariada (p) }\end{array}$ & $\begin{array}{c}\text { Análise } \\
\text { multivariada (p) }\end{array}$ & $\begin{array}{c}\text { Análise } \\
\text { univariada (p) }\end{array}$ & $\begin{array}{c}\text { Análise } \\
\text { multivariada (p) }\end{array}$ \\
\hline Idade & $\overline{0,002528}$ & 0,131775 & 0,181633 & - \\
\hline Sexo & 0,008052 & 0,077717 & 0,820283 & - \\
\hline Inst. públicas & 0,812076 & - & 0,994218 & - \\
\hline Inst. privadas & 0,691254 & - & 0,993733 & - \\
\hline Inst. ensino & 0,273461 & - & 0,994605 & - \\
\hline Consultório & 0,127174 & - & 0,993431 & - \\
\hline Tempo trabalho no posto atual & 0,050526 & 0,685583 & 0,300655 & - \\
\hline Pacientes/dia & 0,013144 & 0,065364 & 0,734533 & - \\
\hline Tempo de consulta & 0,674808 & - & 0,566192 & - \\
\hline Hobby/At. física & 0,285203 & - & 0,241586 & - \\
\hline Apoiofamiliar & 0,041164 & 0,054547 & 0,641355 & - \\
\hline Carga horária & 0,147319 & - & 0,104353 & - \\
\hline Plantões & 0,219942 & - & 0,682990 & - \\
\hline Férias & 0,291043 & - & 0,135070 & - \\
\hline
\end{tabular}

\begin{tabular}{|c|c|}
\hline \multicolumn{2}{|l|}{ Tabela 5 - Resultados do questionário de opinião } \\
\hline & $\mathbf{N}(\%)$ \\
\hline $\begin{array}{l}\text { 1. Limitar o número máximo de pacientes atendidos por dia } \\
\text { 2. Maior tempo gasto em cada consulta } \\
\text { 3. Maior quantidade e qualidade em formação continuada } \\
\text { 4. Relação mais fluida e eficaz com a atenção primária } \\
\text { 5. Menos burocracia } \\
\text { 6. Auxiliares mais colaborativos } \\
\text { 7. Melhor formação sobre a relação médico-paciente } \\
\text { 8. Formação sobre técnicas de relaxamento e canalização do estresse } \\
\text { 9. Objetivos realistas e claros por parte da empresa ou instituição } \\
\text { 10. Definição clara de objetivos e funções de cada membro da equipe }\end{array}$ & $\begin{array}{l}97(73,5 \%) \\
49(37,1 \%) \\
69(52,3 \%) \\
44(33,3 \%) \\
96(72,7 \%) \\
67(50,8 \%) \\
51(38,6 \%) \\
60(45,4 \%) \\
63(47,7 \%) \\
54(40,9 \%)\end{array}$ \\
\hline
\end{tabular}

Fonte: Gonzalez RP, Gonzalez JFS. Prevalecía del Síndrome de Burnout o Desgaste Profesional en los Médicos de Atención Primaria. Aten Primaria, 1998 Nov, 22 (9): 580-4

Uma outra limitação de nosso estudo foi a não caracterização de cada cancerologista quanto à área específica de sua atuação como, por exemplo, oncologia clínica, cirurgia oncológica ou radioterapia. Infelizmente, dada à característica de anonimato das respostas, não se pôde obter este dado após o recebimento das respostas. Acreditamos, entretanto, que este estudo reflete as características da síndrome de exaustão no universo mais amplo de todos os tipos de profissionais brasileiros que se dedicam à cancerologia, e, em um estudo posterior em fase de planejamento, avaliaremos subespecialidades específicas separadamente dentro da cancerologia.

Não há na literatura um consenso para a definição da síndrome da estafa profissional a partir dos valores encontrados no questionário Maslach. A maior parte dos autores descreve os níveis obtidos nas três diferentes dimensões, porém sem um critério definido para agrupá-las. Desta forma, a definição de estafa profissional é confundida com as próprias dimensões. Segundo Grunfeld et al., a síndrome é caracterizada pela presença de cansaço emocional ou despersonalização altos ou realização pessoal baixa. De acordo com Ramirez, apenas os indivíduos que apresentam as três dimensões características do desgaste profissional podem ser considerados portadores da síndrome ${ }^{3,7}$. Assim, apesar de o questionário Maslach ser específico para o diagnóstico da síndrome, a falta de padronização para sua avaliação dificulta a interpretação dos resultados. Em nosso estudo, encontramos 34, I\% de cansaço emocional grave, valor intermediário aos da literatura (25\% e $53,3 \%)^{3,5,7}$. A despersonalização grave obtida em nossa amostra (36,7\%) encontra-se acima da observada em outros estudos nos quais variou de $15 \%$ a 22, $1 \%$. Quanto ao baixo nível de realização pessoal, 
Giglo AD et AL,

outros estudos indicam taxas de 34\% e 48,4\%, superiores à encontrada em nossa amostra (8,6\%).

Os dados demográficos encontrados em nosso estudo são similares aos da literatura. Correlacionando-os com os resultados do questionário Maslach, foi observada significância estatística entre a prática de atividade física ou hobby e menores níveis no escore de exaustão emocional $(p=0,008)$, o que pode ser compreensível dada à sabida importância da atividade física e prática de hobby para a qualidade de vida. Em relação ao local de trabalho, foi encontrada correlação significativa estatisticamente entre trabalhar exclusivamente em instituições públicas e menores níveis de estafa emocional ( $p=0,0$ I2). Este dado não foi encontrado na literatura, porém, inferimos que para estes profissionais seja gratificante trabalhar com alunos, no âmbito de hospitais e instituições públicas, e que estas instituições sejam locais que oferecem menor burocracia e maior liberdade de criaçãa para os profissionais.

Considerando o critério de Grunfeld, encontramos correlação estatisticamente significante entre sexo feminino e ausência de apoio familiar com maiores níveis da síndrome, achados similares aos da literatura ${ }^{6,16}$. Um estudo randomizado realizado com médicos reportou maiores taxas de estafa profissional no sexo feminino, com diminuição significante da síndrome nas profissionais que recebiam apoio familiar ou de amigos ${ }^{16}$.

Em nosso estudo, encontramos uma prevalência muito inferior da síndrome da estafa profissional utilizando o critério de Ramirez quando comparada à encontrada com outras formas de avaliação. Assim, não houve correlações significantes entre a síndrome e as variáveis estudadas, considerando este critério.

Em relação ao questionário de opinião, os resultados obtidos foram condizentes com o encontrado na literatura, em estudo de Gonzalez. Neste estudo, os autores encontraram como mais relevantes para médicos as alternativas: menos burocracia $(72,4 \%)$, limitação do número de pacientes atendidos por dia (72,4\%), maior quantidade e qualidade em formação continuada $(57,6 \%)^{5}$.

\section{Conclusão}

Concluímos que a síndrome da estafa profissional é freqüente entre cancerologistas em nosso meio. Acreditamos que a prevenção desta síndrome seja fundamental para a manutenção da qualidade do atendimento dos pacientes com câncer no Brasil e que futuros estudos devam ser conduzidos para confirmar estes dados e estendê-los também a outras especialidades médicas.

\section{Conflito de interesse: não há.}

\section{SUMMARY}

INCIDENCE OF THE BURNOUT SYNDROME AMONG BRAZILIAN CANCER PHYSICIANS

BACKGROUNDS. The Burnout syndrome can be characterized by abnormalities in three domains: emotional exhaustion (EE), depersonalization (DP) and lack of personal realization (PR). In the medical profession, oncologists are especially prone to this syndrome. This study evaluates its prevalence among cancer physicians in Brazil correlating it to their demographic, work related variables and seeks possible solutions to prevent burnout.

Methods. We mailed three questionnaires (Maslach burnout inventory, general and opinion questionnaires) to all 645 members of the Brazilian Cancer Society and received I 36 responses after 10 weeks.

RESULTS. The response rate was of $21 \%$. The burnout syndrome was present at moderate or severe levels in all three domains analyzed in $15.7 \%$ of the physicians. For each of these the frequency of moderate or severe dysfunction was analyzed and found to be present in $55.8 \%$ for EE, 96. $1 \%$ for DP and $23.4 \%$ for RP. Practicing physical activity or having a hobby correlated significantly with lower levels of EE $(\mathrm{p}=0.008)$ while working only for the private sector correlated with higher DP scores $(\mathrm{p}=0.021)$. Cancer physicians pointed out that less paper work $(73.5 \%)$ and a lower patient load (72.7\%) were the most important factors for prevention of this syndrome.

CONCLUSION. Burnout syndrome is frequent among Brazilian cancer physicians and further studies should be conducted to evaluate its prevalence and prevention among other sub specialists. [Rev Assoc Med Bras 2006; 52(2): 108-12]

KeY words: Professional burnout. Medical oncology. Depersonalization.

\section{REFERÊNCIAS}

I. Carlotto MS, Gobbi MD. Síndrome de Burnout: um problema do indivíduo ou do seu contexto de trabalho? Aletheia 1999; 10:103-14.

2. Gatto ME. Desgaste psíquico en el equipo de salud. Síndrome de Burnout. Pren Méd Argent 2000; 87: 357-6I

3. Ramirez AJ, Graham J, Richards MA, CuffA, Gregory WM, Leaning MS, et al. Burnout and psychiatric disorder among cancer clinicians. Br J Cancer 1995,71: | 263-69.

4. Muñoz AJJ. El trastorno por estrés postraumático en oncología: Abordaje clínico y manejo de estrés en el equipo médico. Rev Sanid Milit Mex 2000;54:93-9.

5. Gonzalez RP, Gonzalez JFS. Prevalencia del Síndrome de Burnout o desgaste professional en los médicos de atención primária. Aten Primaria 1998; 22:580-4.

6. Gabbe SG, Melville J, Mandel L, Walker E. Burnout in chairs of obstetrics and gynecology: diagnosis, treatment, and prevention. Am J Obstet Gynecol 2002; | 86:60 I - 12.

7. Grunfeld E, Whelan T], Zitzelsberger L, Willan AR, Montesanto B, Evans WK. Cancer care workers in Ontario: prevalence of burnout, job stress and job satisfaction. CMAJ 2000; 1 63: I 66-9.

8. Linzer M, Visser MRM, Oort FJ, Smets EMA, McMurray JE, Haes HCJM. Predicting and preventing physician burnout: results from the United States and the Netherlands. Am | Med 2001; I I : 170-5.

9. Souza GF, Baptista NM, Xidieh FG. Burnout: definição e característica. Infanto. Rev Neuropsiquiatr Infanc Adolesc 200 I;9: 100-2.

10. Tena SP, Soriano SJ, Bernal SJ, Mulet ME, Muñoz RC, García GM, et al. Desgaste profesional en los médicos de Atención Primaria de Barcelona. Medifam 2002; I 2(I0)

1 I. Gil-Monte RP. Validez factorial de la adaptación al español del Maslasch Burnout Inventory: general survey. Salud Pública Mex 2002;44:33-40.

12. Creagan ET. Stress among medical oncologists: the phenomenon of burnout and a call to action. Br I Cancer 1994:70:767-70.

13. Borges LO, Argolo TJC, Pereira SAL, Machado PEA, Silva SW. A Síndrome de Burnout e os valores organizacionais: um estudo comparativo em hospitais universitários. Psicol Reflex Crit 2002; I 5: I 89-200.

14. Olivar CC, Gonzalez MS, Martinez SMM. The factors related to job satisfaction and professional burn in the primary care physicians of Asturias. Aten Primaria 1999; 24:352-9.

15. Whippen DA, Canellos JP. Syndrome in the practice of Oncology: Results of a Randon Survey of 1000 Oncologists. J Clin Oncol 1991;9:1916-20.

16. McMurray JE, Linzer M, Konrad TR, Douglas J, Shugerman R, Nelson K. The work lives of women physicians results from the physician work life study. J Gen Intern Med 2000; 1 5:372-80.

Artigo recebido: 24/I I/04

Aceito para publicação: 28//2/05 